\title{
The Alveolar Stem Cell Niche of the Mammalian Lung
}

\author{
Brigid L. M. Hogan
}

\begin{abstract}
The alveolar region of the mammalian lung evolved to enable highly efficient gas exchange between the millions of air-filled sacs known as alveoli and blood circulating through the pulmonary vessels. Each alveolus is composed of epithelial and mesenchymal populations; Type 1 (AT1) and Type 2 (AT2) epithelial cells line the sacs while the mesenchymal compartment is composed of endothelial cells, pericytes, several different fibroblast subpopulations, and immune cells. Compared with organs such as the intestine and skin, there is normally little cell turnover in the adult lung. However, if the alveolar epithelium is damaged, for example, by toxic agents or viral infections, or if a lung lobe is removed, there is extensive repair or compensatory regrowth (neoalveolarization) of the tissue. It is now well accepted that AT2s function as epithelial stem cells for the alveoli, capable of long-term self-renewal and differentiation into AT1s. However, many important questions remain, in particular, about the functional heterogeneity of AT2s and how the different components of their local environment or niche interact to regulate their proliferation and differentiation. Known signaling factors include ligands and antagonists of the WNT, FGF, EGF and bone morphogenetic (BMP) signaling pathways as well as cytokines produced by immune cells. The relative contribution of these factors to alveolar homeostasis, repair and regrowth is discussed.
\end{abstract}

\section{Keywords}

Lung $\cdot$ Alveolar stem cell niche $\cdot$ Compensatory regrowth $\cdot$ Signaling pathways

B. L. M. Hogan $(\bowtie)$

Department of Cell Biology, Duke University Medical School, Durham, NC, USA

e-mail: brigid.hogan@duke.edu 


\subsection{Introduction: The Alveolar Type 2 Epithelial Stem Cell Niche}

Our current understanding of the alveolar stem cell niche of the mammalian lung is schematized in Fig. 2.1 [1-4]. Each alveolus contains two kinds of epithelial cells: Type 2 cells (AT2s), specialized for producing, secreting and recycling surfactant lipids and proteins, and Type 1 cells (AT1s) that are extremely large and thin and specialized for gas exchange [5]. It is now well accepted AT2s that express surfactant protein $\mathrm{C}$ ( $\mathrm{Sftpc}$ ) can function as stem cells during homeostasis and repair by self-renewing and giving rise to AT1s. However, the rate at which AT2s proliferate and differentiate depends on local "demand." At steady state the rate is very low. If lungs are experimentally depleted of only AT2s then their proliferation increases but differentiation is still minimal [6]. However, if AT1s are also damaged or if whole new alveoli need to be generated in response to partial (left lobe) pneumonectomy (PPnx), then AT2s both self-renew and show robust AT1 differentiation [7-9]. How AT2s detect the absence of AT1s is unclear; perhaps they sense denuded basal lamina or a reduction in cellular contacts with AT1s or, in the case of PPnx, an increase in mechanical tension [10].

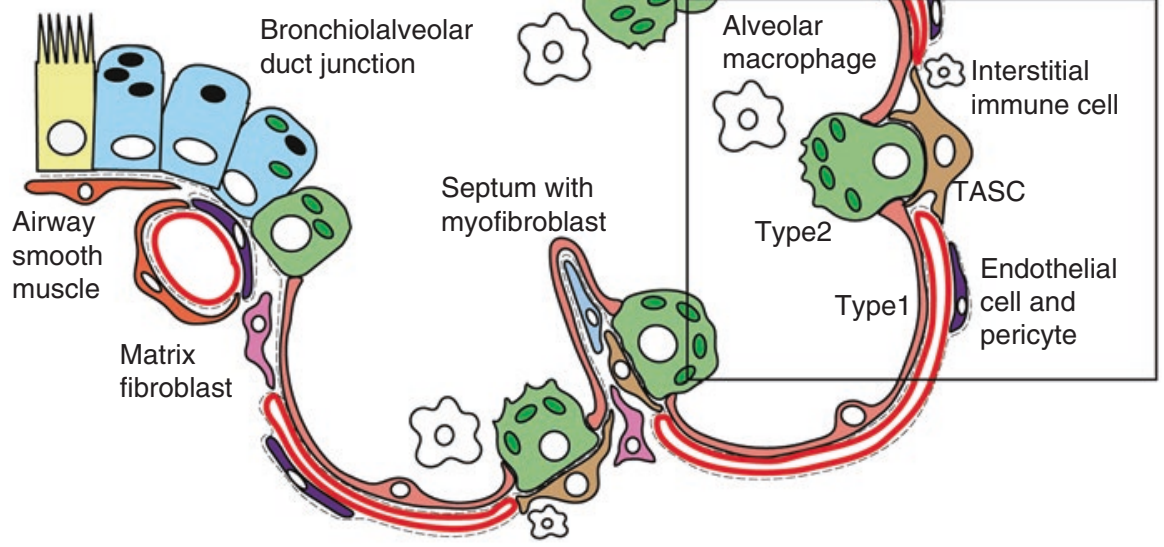

Fig. 2.1 Schematic representation of the alveolar region of the mouse lung. The Type 2 stem cell niche (boxed) includes fibroblasts known as TASCs that have long cytoplasmic extensions, AT1 cells, capillary endothelial cells and pericytes as well as alveolar macrophages and interstitial immune cells. Alveolar septae contain myofibroblasts, and other fibroblast cell types (matrix fibroblasts) are present in the stroma 


\subsection{Evidence for Heterogeneity in the AT2 Population}

An important unanswered question is whether all AT2s have the same stem cell potential or whether this is a property of a privileged few. Significantly, two groups have recently shown that at steady state a minority of AT2s express a reporter for Axin2, a downstream target of canonical Wnt signaling. One group [11] found that only $1 \%$ of the AT2s were positive although more cells became active in response to injury. By contrast, a second group [12] reported that 20-30\% of AT2s were positive at steady state and constituted a stable subpopulation which they termed alveolar epithelial progenitors (AEPs). In both cases evidence was presented that the Wnt ligand responsible for upregulating Axin2 and promoting AT2 proliferation is produced by niche fibroblasts in close proximity to AT2s, termed MANCs (mesenchymal alveolar niche cells). These cells can also produce Wnt antagonists, which reduce AT2 proliferation and promote differentiation, and may, therefore, fine tune the dynamic response of the stem cells during repair [13].

In the light of these studies, one may ask whether AT2 subpopulations have been detected by single-cell transcriptomic analysis. One recent study does show a minor AT2 subpopulation in the steady state lung, defined as "alveolar bipotential progenitors" [14]. However, these cells are distinguished by co-expressing markers of AT2s and AT1s but not by Axin2 levels. In support of the single-cell RNA-seq data, a recent report [8] identified by immunohistochemistry a small subpopulation (less than 1\%) of AT2s co-expressing Sftpc and Ager (advanced glycosylation and end product-specific receptor, a marker for AT1s). The proportion of these dual-positive cells increases to $20 \%$ by 7 days after PPnx when compensatory cell proliferation is active. In addition, when isolated AT2 cells are placed in 3D organoid culture under conditions in which single cells give rise to structures known as "alveolospheres" containing both AT2s and AT1s, all of the cells initially and transiently become dual positive for Sftpc and Ager [8]. This behavior may indicate a reversion of mature AT2s to a more plastic, bipotential state under conditions promoting repair. New genetic tools are clearly needed to test the in vivo significance of the dual-positive cells and their relation, if any, to Axin2+ epithelial cells [11, 12].

\subsection{Signaling Pathways in the Stem Cell Niche}

Besides WNTs and their antagonists, a number of other signaling factors have been identified as playing a role in the mouse lung alveolar stem cell niche. Pathways include those regulated by Fgfs (largely produced by fibroblast populations), Vegf (secreted by AT2s), Bmps and Egfs. Egf signaling is thought to be mediated both by the ligand itself and by peptides released from the extracellular matrix after injury. This latter mechanism has been proposed for the proliferation of AT2s after PPnx [15]. Specifically, it appears that in response to PPnx pulmonary capillary endothelial cells (PCECs) activate the expression of the matrix metalloproteinase MMP14. This degrades laminin and releases peptides with a cryptic Egf domain that can bind to Egf receptors in the AT2 cells. Changes in PCECs and perivascular macrophages 
have also been implicated in the response of the alveolar region to damage by bleomycin or HCL [16]. In this case, it is argued that the notch ligand Jagged1 is upregulated in the damaged PCECs, affecting the behavior of adjacent fibroblasts. Given the recent finding that lung microvasculature is a major site of platelet biogenesis and a reservoir for hematopoietic progenitors [17] there are likely many other ways in which cytokines and angiocrine factors produced by the vascular compartment influence the stem cell niche.

BMP signaling in the alveolar niche has not received much attention until recently since most research has focused on the pathway in the etiology of pulmonary hypertension. However, immunohistochemistry with antibodies to phosphoSmad1/5/8 shows that BMP signaling is active in both epithelial and stromal cells of the alveolar region. Signaling is transiently downregulated after PPnx when AT2s are proliferating and preparing to differentiate into AT1s. This transient downregulation is likely brought about by the upregulation of BMP antagonists, including follistatin and follistatin-like 1 by stromal cells rather than downregulation of BMP ligands [8].

\subsection{The Role of Immune Cells and Stromal Cells in Alveolar Repair and Regeneration}

One of the major roadblocks to our understanding of the alveolar stem cell niche is the precise identification of the different mesenchymal/mesoderm-derived cell types in the region. This population includes lipofibroblasts (cells with varying numbers of lipid inclusion), myofibroblasts (cells rich in smooth muscle actin) and "matrix fibroblasts" and their progenitors $[13,14,18]$. The lung also contains a rich and varied population of resident immune cells with multiple subclasses of dendritic cells and innate lymphoid cells, and interstitial and alveolar macrophages. Immune cells are recruited to the alveolar niche after injury and can play important roles in repair and regrowth. Recent studies have shown a role for bone marrow-derived macrophages in compensatory regrowth after PPnx [9]. Genetic deletion of the bone marrow-derived macrophages, or the innate immune cells thought to activate them, results in reduced AT2 proliferation and regrowth. In the future, it will be important to determine the precise mechanism by which the macrophages promote repair, for example, whether they produce cytokines acting directly on AT2s or function indirectly through other components of the niche.

Progress in defining the precise physiological function of the different stromal populations in the alveolar region has been hampered by the relative paucity of tools for genetically manipulating and lineage tracing specific cell types. We, therefore, generated a new Pdgfra-CreEr knock-in allele (Jackson Lab stock number 032770) to test the function of genes expressed in fibroblasts expressing the Pdgf receptor alpha. This is a heterogeneous population but includes cells intimately associated with AT2s that we have called Type 2 associated stromal cells, or TASCs [8]. These cells have a very distinct morphology with long cellular extensions and may be the same as the MANCs described by others [13]. They likely represent the mouse 
equivalent of cells described in the human lung that make contact with AT2, AT1 and endothelial cells [19] and are, therefore, well positioned to integrate signaling pathways within the niche.

\subsection{Future Directions and Clinical Implications}

This review highlights recent advances in our understanding of the cells and signaling pathways that regulate AT2 proliferation and differentiation in the alveolar niche of the mouse lung. In the long term, this information may inspire new clinical strategies for promoting alveolar repair from remaining endogenous stem cells in damaged human lungs. A major challenge is to translate techniques used with mouse cells to primary cells isolated from the human lung. For example, the "alveolosphere" organoid culture system that has been used so successfully to screen for factors that regulate AT2 stem cell behavior is much less efficient with human AT2 cells for reasons that are currently unclear [20]. Nevertheless, progress is being made and we should expect to see important advances in the near future.

Acknowledgment Work from the authors' laboratory was funded by the USA National Heart, Lung and Blood Institute.

\section{References}

1. Leach JP, Morrisey EE. Repairing the lungs one breath at a time: how dedicated or facultative are you? Genes Dev. 2018;32(23-24):1461-71.

2. Hogan BL, et al. Repair and regeneration of the respiratory system: complexity, plasticity, and mechanisms of lung stem cell function. Cell Stem Cell. 2014;15(2):123-38.

3. Tata PR, Rajagopal J. Plasticity in the lung: making and breaking cell identity. Development. 2017;144(5):755-66.

4. Borok Z, et al. Cell plasticity in lung injury and repair: report from an NHLBI workshop, April 19-20, 2010. Proc Am Thorac Soc. 2011;8(3):215-22.

5. Weibel ER. On the tricks alveolar epithelial cells play to make a good lung. Am J Respir Crit Care Med. 2015;191(5):504-13.

6. Barkauskas CE, et al. Type 2 alveolar cells are stem cells in adult lung. J Clin Invest. 2013;123(7):3025-36.

7. Desai TJ, Brownfield DG, Krasnow MA. Alveolar progenitor and stem cells in lung development, renewal and cancer. Nature. 2014;507(7491):190-4.

8. Chung MI, et al. Niche-mediated BMP/SMAD signaling regulates lung alveolar stem cell proliferation and differentiation. Development. 2018;145(9)

9. Lechner AJ, et al. Recruited monocytes and Type 2 immunity promote lung regeneration following pneumonectomy. Cell Stem Cell. 2017;21(1):120-134.e7.

10. Liu Z, et al. MAPK-mediated YAP activation controls mechanical-tension-induced pulmonary alveolar regeneration. Cell Rep. 2016;16(7):1810-9.

11. Nabhan AN, et al. Single-cell Wnt signaling niches maintain stemness of alveolar type 2 cells. Science. 2018;359(6380):1118-23.

12. Zacharias WJ, et al. Regeneration of the lung alveolus by an evolutionarily conserved epithelial progenitor. Nature. 2018;555(7695):251-5. 
13. Zepp JA, et al. Distinct mesenchymal lineages and niches promote epithelial self-renewal and myofibrogenesis in the lung. Cell. 2017;170(6):1134-1148.e10.

14. Han X, et al. Mapping the mouse cell atlas by Microwell-Seq. Cell. 2018;172(5):1091-1107. e17.

15. Ding BS, et al. Endothelial-derived angiocrine signals induce and sustain regenerative lung alveolarization. Cell. 2011;147(3):539-53.

16. Cao Z, et al. Targeting of the pulmonary capillary vascular niche promotes lung alveolar repair and ameliorates fibrosis. Nat Med. 2016;22(2):154-62.

17. Lefrancais E, et al. The lung is a site of platelet biogenesis and a reservoir for haematopoietic progenitors. Nature. 2017;544(7648):105-9.

18. Tabula Muris $\mathrm{C}$, et al. Single-cell transcriptomics of 20 mouse organs creates a Tabula Muris. Nature. 2018;562(7727):367-72.

19. Sirianni FE, Chu FS, Walker DC. Human alveolar wall fibroblasts directly link epithelial type 2 cells to capillary endothelium. Am J Respir Crit Care Med. 2003;168(12):1532-7.

20. Barkauskas CE, et al. Lung organoids: current uses and future promise. Development. 2017;144(6):986-97.

Open Access This chapter is licensed under the terms of the Creative Commons Attribution 4.0 International License (http://creativecommons.org/licenses/by/4.0/), which permits use, sharing, adaptation, distribution and reproduction in any medium or format, as long as you give appropriate credit to the original author(s) and the source, provide a link to the Creative Commons license and indicate if changes were made.

The images or other third party material in this chapter are included in the chapter's Creative Commons license, unless indicated otherwise in a credit line to the material. If material is not included in the chapter's Creative Commons license and your intended use is not permitted by statutory regulation or exceeds the permitted use, you will need to obtain permission directly from the copyright holder.

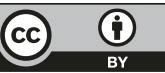

